\title{
Design of Post-Wall Waveguides with Metal and Dielectric Posts
}

\author{
T.J. Coenen ${ }^{* 1}$, D.J. Bekers* ${ }^{*}$ J.L. Tauritz ${ }^{\dagger}$, F.E. van Vliet* \\ *TNO Defence, Security and Safety \\ P.O. Box 96864, 2509 JG Den Haag, The Netherlands \\ ${ }^{1}$ teis.coenenetno.nl \\ ${ }^{\dagger}$ Department of Telecommunication Engineering, University of Twente \\ P.O. Box 217, 7500 AE Enschede, The Netherlands
}

\begin{abstract}
Post-wall waveguides can fulfill the requirements for low-loss transmission lines and components in the interconnection between antenna and front-end electronics. In this paper we present a model based on an element-by-element method for simulating wave propagation in metallic and dielectric post-wall waveguides. We demonstrate that both types of waveguides support wave propagation and we show that undesired resonances can limit the range of operation.
\end{abstract}

\section{INTRODUCTION}

With the increase in working frequency of current microwave systems to the millimetre wave regime, the need for novel and low-loss transmission lines and components in implementing interconnects between antenna and front-end electronics is manifest. Preferably these structures should be low cost, integrable and easy to manufacture. Post-wall waveguides can satisfy these three requirements and serve as low-loss building blocks for an antenna feed network.

A post-wall waveguide consists of a dielectric slab with metalization at the top and bottom. Within the slab linear arrays of cylindrical posts, bound an interior waveguiding region as depicted in Fig. 1. The posts can be metalized vias

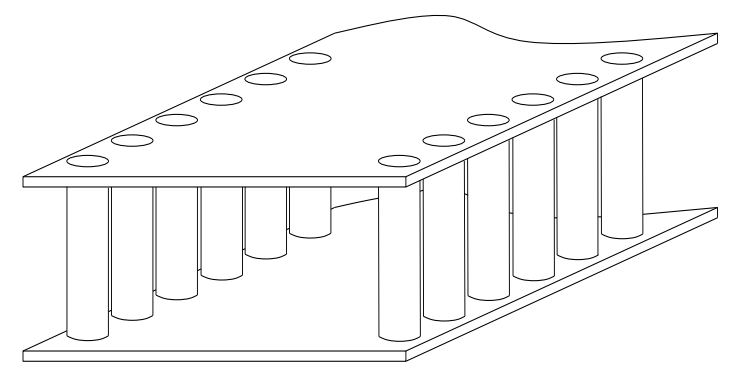

Fig. 1. Basic impression of a post-wall waveguide.

or drilled holes in PCB dielectric material. Both structures are relatively easy to manufacture and low cost.

To date, most of the work on post-wall waveguides, is devoted to analysis and design with metallic posts [1], [2]. For an overview of analysis techniques for such post-wall waveguides, such as BI-RME, transversal resonance technique, FDFD, we refer to [3]. The dielectric post-wall waveguide is not extensively treated in literature. Examples are the post-wall waveguide with low dielectric material on top and bottom, as presented in [4], and the variation on the dielectric image line, with a metal ground plane on the bottom, as presented in [5], [6]. These guides are designed using brute-force numerical tools based on FEM or FDTD solvers. Our strategy is to analyze both metallic and dielectric post-wall waveguides using a dedicated Method of Moments analysis while taking into account the physics of the problem. In [7], we demonstrated that wave propagation in a dielectric post-wall waveguide is possible, where we used a simplified model specifically suited for posts of small diameter (typically smaller than $\lambda / 8$ ). In this paper we describe a general method of analysis for both metallic and dielectric post-wall waveguides. Moreover, we compare post-wall waveguides of metallic and dielectric posts, where the diameter of the dielectric posts is larger to ensure better confinement of the field. Finally we propose test structures for validating our analysis.

\section{MODEL}

Fig. 2 shows the wave propagation in a post-wall waveguide with side walls consisting of more than one linear array. We model this propagation as a plane wave reflecting at the side walls by imposing appropriate boundary conditions. Moreover, we assume that

1) the cylinders are homogeneous,

2) the cylinders extend infinitely in the axial direction, and

3) only an electric field in the axial direction exists.

This implies that the field components in the axial direction only vary by a phase term reducing in effect to a $2 \mathrm{D}$ problem with the electric field solely described by the axial component. As a consequence, wave propagation can only occur in the $(x, y)$-plane.

Using a similar approach as in [8], we expand the excitation field $E_{z}^{\text {exc }}$, the scattered field $E_{z}^{\text {sct }}$ and the field inside the 


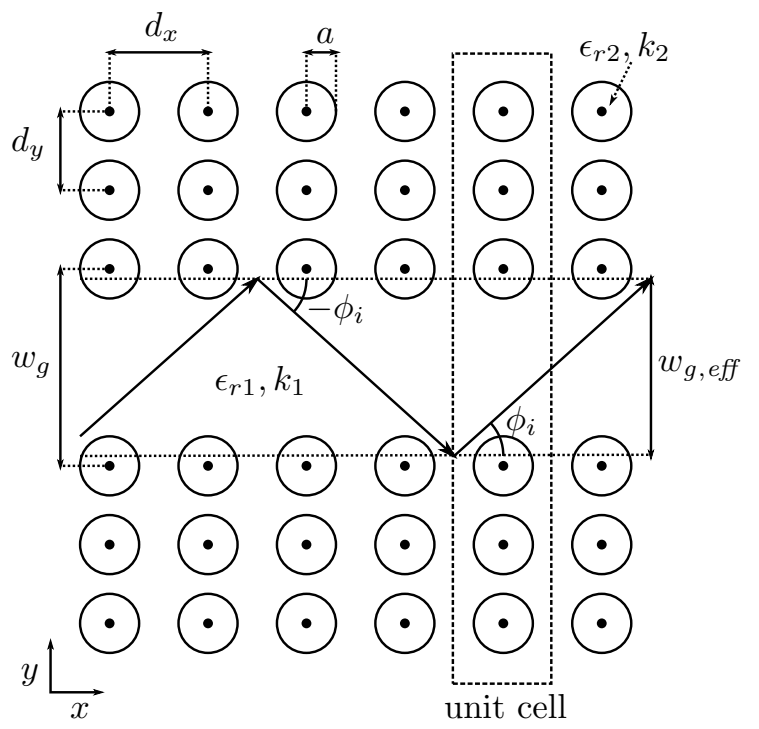

Fig. 2. Geometry of a post-wall waveguide (top view).

cylinder $E_{z}^{\text {int }}$ by Fourier series

$$
\begin{aligned}
& E_{z}^{\mathrm{exc}}=E_{0} \sum_{m=-\infty}^{\infty} j^{-m} J_{m}\left(k_{1} r\right) e^{j m\left(\phi-\phi_{i}\right)} \\
& E_{z}^{\mathrm{sct}}=E_{0} \sum_{m=-\infty}^{\infty} A_{m} j^{-m} H_{m}^{(2)}\left(k_{1} r\right) e^{j m \phi} \\
& E_{z}^{\mathrm{int}}=E_{0} \sum_{m=-\infty}^{\infty} B_{m} j^{-m} J_{m}\left(k_{2} r\right) e^{j m \phi}
\end{aligned}
$$

and we enforce the continuity of the tangential electric (and magnetic) fields on the cylinder boundaries. This leads to a matrix systems of equations

$$
\left[\begin{array}{cccc}
{\left[\mathbf{C}_{11}\right]} & {\left[\mathbf{C}_{12}\right]} & \cdots & {\left[\mathbf{C}_{1 \mathbf{P}}\right]} \\
{\left[\mathbf{C}_{\mathbf{2 1}}\right]} & {\left[\mathbf{C}_{22}\right]} & & \\
\vdots & & \ddots & \\
{\left[\mathbf{C}_{\mathbf{P 1}}\right]} & & & {\left[\mathbf{C}_{\mathbf{P P}}\right]}
\end{array}\right]\left[\begin{array}{c}
\mathbf{A}_{1} \\
\mathbf{A}_{2} \\
\vdots \\
\mathbf{A}_{\mathbf{P}}
\end{array}\right]=\left[\begin{array}{c}
\boldsymbol{\Phi}_{1} \\
\boldsymbol{\Phi}_{2} \\
\vdots \\
\boldsymbol{\Phi}_{\mathbf{P}}
\end{array}\right]
$$

where the A's are the unknown field expansion coefficients from (2) and $\boldsymbol{\Phi}$ is a phase term

$$
\Phi_{p, m}=-j^{m} e^{-j m \phi_{i}} e^{j k_{1} r_{0 p} \cos \left(\phi_{0 p}-\phi_{i}\right)}
$$

In the case of dielectric cylinders the elements of the block matrices $\mathbf{C}_{\mathbf{P Q}}$ can be written as

$$
\begin{aligned}
& C_{p q, m n}= \\
& \left\{\begin{array}{lc}
0 & (p=q, \\
& m \neq n) \\
\frac{k_{2} H_{m}^{(2)}\left(k_{1} a\right) J_{m}^{\prime}\left(k_{2} a\right)-k_{1} H_{m}^{\prime(2)}\left(k_{1} a\right) J_{m}\left(k_{2} a\right)}{k_{2} J_{m}\left(k_{1} a\right) J_{m}^{\prime}\left(k_{2} a\right)-k_{1} J_{m}^{\prime}\left(k_{1} a\right) J_{m}\left(k_{2} a\right)} & (p=q, \\
& m=n) \\
H_{m-n}^{(2)}\left(k_{1} r_{p q}\right) e^{-j(m-n) \phi_{p q}} & (p \neq q)
\end{array}\right.
\end{aligned}
$$




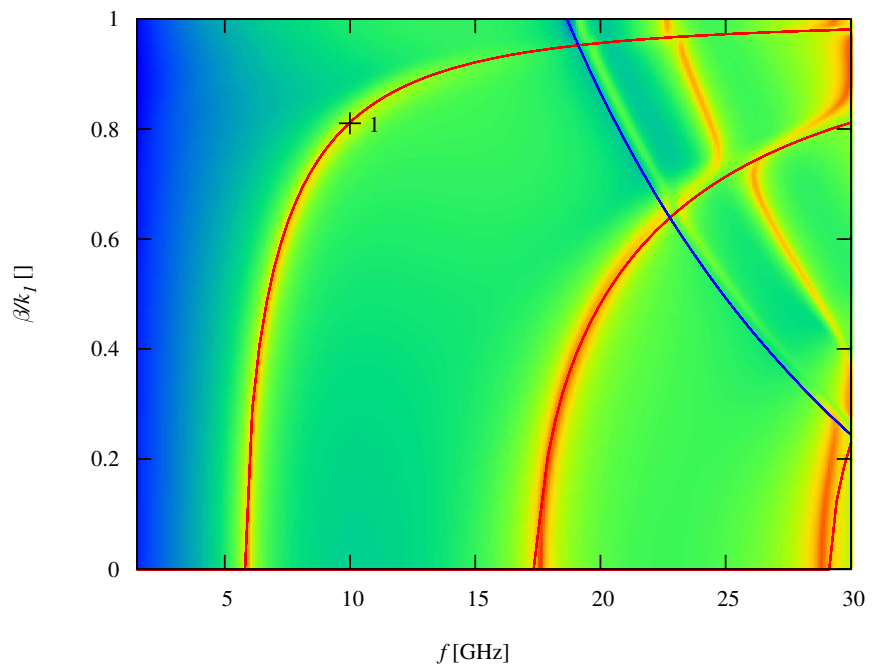

(a) Metallic posts

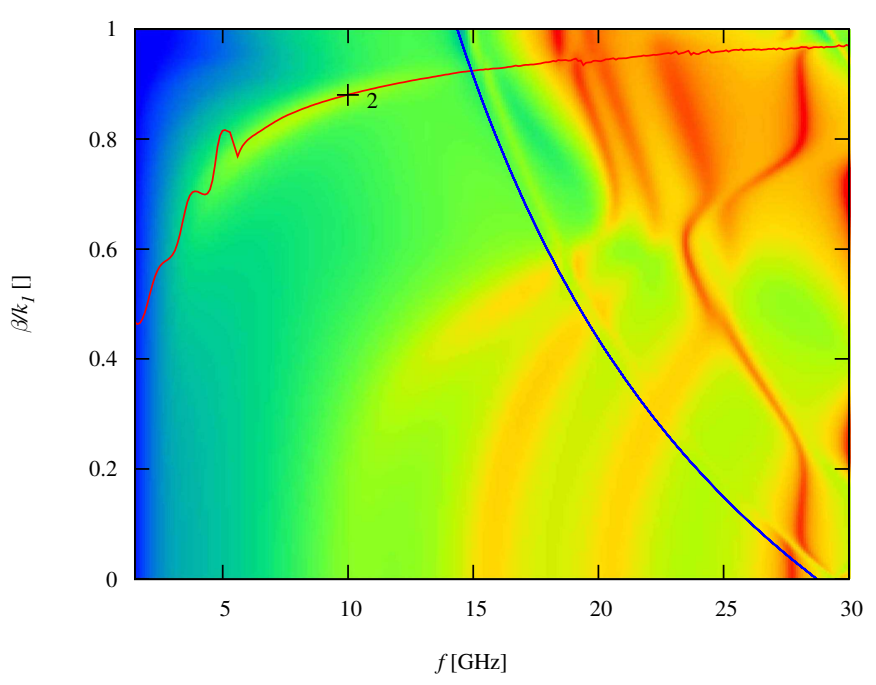

(b) Dielectric posts

Fig. 3. Modal resonances for a post-wall waveguide with metallic cylinders compared with a rectangular waveguide and a post-wall waveguide with dielectric cylinders compared with HFSS results.

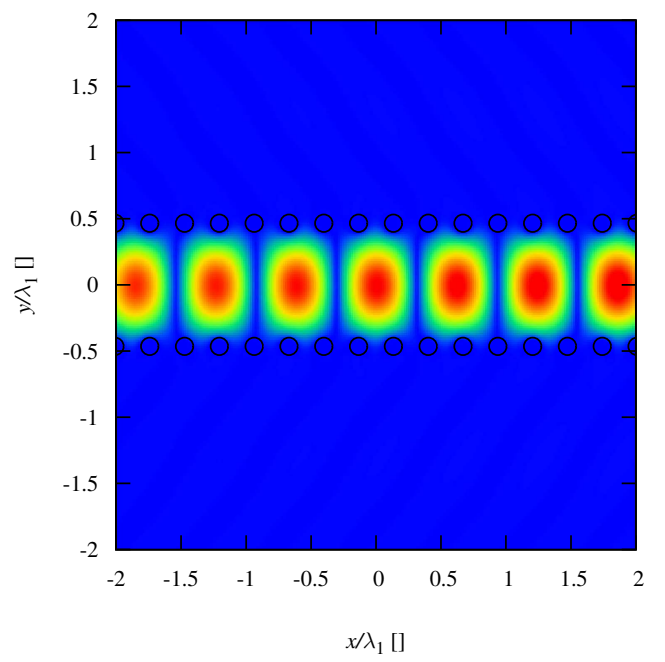

(a) Marker 1 in Fig. 3(a)

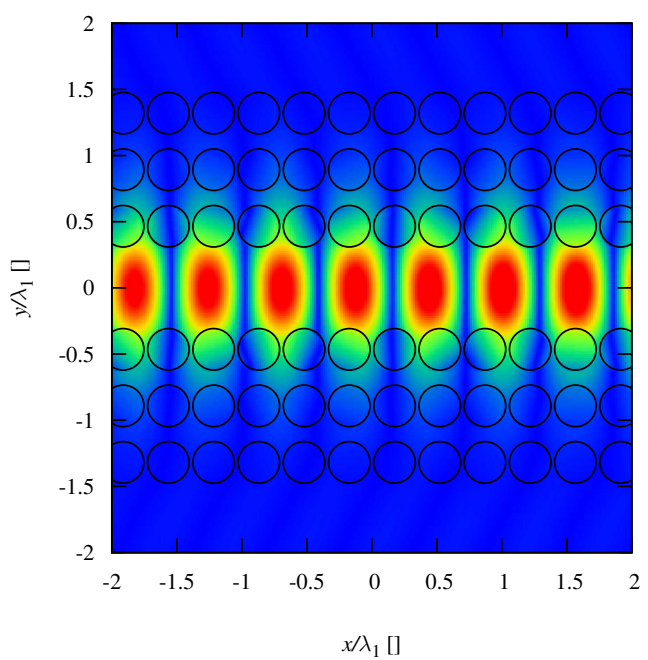

(b) Marker 2 in Fig. 3(b)

Fig. 4. Plots of $\left|\Re\left\{E_{z}\right\}\right|$ at $f=10 \mathrm{GHz}$ for the dominant mode in a post wall waveguide with metallic (a) and dielectric (b) posts.

dispersion curves match two clearly dinstinguishable curves in the color plot. This observation illustrates the correspondence between wave propagation in classical rectangular waveguides and post-wall waveguides. Moreover, wave propagation can indeed be identified from the color scale in Fig. 3(a). For the dielectric case a comparison with simulation results from Ansoft HFSS is shown (red lines). As in the metallic case, these results match a clearly distinguishable curve in the color plot of Fig. 3(b). Fig. 4(a) and 4(b) depict the electric field in the $(x, y)$-plane for the main propagating mode, as indicated by marker 1 and 2 in Fig. 3(a) and 3(b), in the metallic and dielectric post-wall waveguide respectively. In the case of the metallic posts we observe that the pattern is similar to that of the same mode in a rectangular waveguide and that the field is well-confined within the waveguide. The field plot for the dielectric posts shows that more parallel arrays of cylinders per side-wall are required to confine the field within the guiding region.

\section{Measurement Results}

In order to compare our analysis and the results from the HFSS simulations with data from measurements we are currently manufacturing a set of post-wall waveguides. The test samples are being fabricated in a microwave laminate, using standard PCB techniques. We use a slot-coupled microstrip line to excite the post-wall waveguide structures. In order to determine the propagation constant of the two different types of post-wall waveguide, we use the multiline method [9]. This method uses redundant measurements of several lengths 
of post-wall waveguide to minimize the error in the determination of the propagation constant. The test structures are representative of the metallic post-wall and dielectric post-wall waveguides discussed in this paper. The measurement results will be presented at the conference.

\section{CONCLUSIONS}

We presented a method for analysis of post-wall waveguides consisting of either metallic or dielectric cylinders and we demonstrated that guided wave propagation occurs in both types of structure. As soon as the first grating-lobe appears, undesirable resonances can occur giving rise to radiation effects that limit guided wave propagation.

Finally, we proposed a set of test structures for which measurement results will be presented at the conference.

\section{REFERENCES}

[1] J. Hirokawa and M. Ando, "Single-layer feed waveguide consisting of posts for plane TEM wave excitation in parallel plates," IEEE Trans. Antennas Propagat., vol. 46, no. 5, pp. 625-630, May 1998.

[2] H. Uchimura, T. Takenoshita, and M. Fujii, "Development of a "Laminated Waveguide"," IEEE Trans. Microwave Theory Techn., vol. 46, no. 12 , pp. 2438-2443, December 1998.

[3] D. Deslandes and K. Wu, "Accurate modelling, wave mechanisms, and design considerations of a substrate integrated waveguide," IEEE Trans. Microwave Theory Techn., vol. 54, no. 6, pp. 2516-2526, June 2006.

[4] I. El-Kady, M. Sigalas, R. Biswas, and K. Ho, "Dielectric waveguides in two-dimensional photonic bandgap materials," J. Lightwave Technol., vol. 17, no. 11, pp. 2042-2049, November 1999.

[5] A. Patrovsky and K. Wu, "Substrate integrated image guide (SIIG) - a low-loss waveguide for millimetre-wave applications," in Proc. 35th Eur. Microw. Conf., Paris, France, October 2005, pp. 897-900.

[6] _ - "Substrate integrated image guide (SIIG) — a planar dielectric waveguide technology for millimeter-wave applications," IEEE Trans. Microwave Theory Techn., vol. 54, no. 6, pp. 2872-2879, June 2006.

[7] T. Coenen, A. Mazzinghi, D. Bekers, A. Neto, J. Tauritz, A. Freni, and G. Gerini, "Design and analysis of EBG based integrated waveguide structures for microwave and MM-wave feed networks," in Proc. IEEE AP-S Int. Symp. Dig., Albuquerque, NM, July 2006, p. 654.

[8] G. Olaofe, "Scattering cross section for two cylinders," IEEE Trans. Antennas Propagat., vol. AP-18, no. 11, pp. 823-825, November 1970.

[9] R. Marks, "A multiline method of network analyzer calibration," IEEE Trans. Microwave Theory Techn., vol. 39, no. 7, pp. 1205-1215, Jul. 1991. 